\title{
Subject Index Vol. 36, 1997
}

Actigraphy 57, 211 Activation 87 Aerobic exercise 182 Affective disorders 164 Agoraphobia 25 Agranulocytosis 5 Alcohol 1

Alzheimer's disease 73 Amantadine 22 AMDP modules 100 Amphetamine 177 Analysis of partial variance 107 Anterograde amnesia 42 Anxiety 164 Arousal 65

Association analysis 62 Asymmetry 22 Auto-immunity 172 Automated scoring 194 Azathioprine 172

Benzodiazepines 25 Biperiden plasma levels 69 Brain damage 1 Brief psychiatric rating scale 32

Calcium 164 Cannabinoid 96 Cannabis 96

Cardiopulmonary fitness 182 Ceruletide 87 Cholecystokinin 87 Chronic schizophrenia 69, 112 Circadian rhythm 159 Clinical drug trials 73 Clozapine treatment 5 Cocaine 53, 83 Comorbid depression 25 Cortisol 8

Delta-9-tetrahydrocannabinol 96 Deoxyglucose 177 Depression 57,107 Dexamethasone suppression test 19 Diazepam 42 Diurnal tiredness 117 - variation of mood 57 Dopamine 1, 96 Drug-resistant schizophrenics 51

Electroencephalographic mapping 117, 130 Electroencephalography 49 Electrolytes 164 Electrophysiology 96 Equal-environment assumption 13

Erythrocytes 164

Ethanol 188

Event-related potential(s) 53, 83

Evolutionary algorithms 194

Extrapyramidal side effects 69

Fast Fourier transformation analysis 153 ú?-Fenfluramine 8

GABRA5 gene 62

Generalized anxiety disorder 117,130

Genetic algorithms 194

Global deterioration scale 153

Goiter 37

Haloperidol 32, 65

- $\quad$ plasma levels 69

Heritability 13

HLA-typing 5

Hypervigilance 117

Hypnotic and anxiolytic effects 130

Idebenone 73 Imipramine 25 Immunology 107

Lithium 37 Lorazepam 130 Lorazepam/diphenhydramine combination 130

Melatonin 159,211

Memory 42

Methylphenidate 42

Mini Mental State examination 153

Mitogen-induced lymphocyte responses 107

Mood disorders 62

Motor activity 57,211

N40 83

Nerve growth factor 65 Neural networks 194 Neuroleptics 159 Neuropsychopharmacology 49 Nonorganic insomnia 117,130 Noopsyche 130 Noopsychic performance 117

Objective signs 57 Obsessive-compulsive disorder 8

P300 53

Panic disorder 25

- - agoraphobia 182

Parasomnia 211

Pituitary-adrenal function 112 
Plasma alpha-one acid glycoprotein 32

cortisol levels 19 Platelet auto-antibodies 172

5-HT concentrations 19 Polysomnography 117,130 Positive and negative symptoms 19 Potassium 164

PRL 1

Processing negativity 87 Psychiatric EEG 100 Psychomotor retardation 164

Rabbit 49

Rat 177

Reinforcement 96

Relation between dose and plasma levels 69

REM sleep 211

Reward 96

Schizophrenia 13, 19, 22, 32, 65, 159, 172

Sedation 65

Selective attention 87

Sensitization 177

Sensory gating 83

Serotonin 8

Severity of disease 73

Sex difference 8

Sleep EEG 194

pressure 117

time 188 Sodium 164 Somnium ${ }^{\circledR} 130$ Standardization 100

Subjective sleep and awakening quality 117,130

symptoms 57

Surgical stress, antipsychotics 112 Symptoms 164

T/C ratio 83 Tetrahydrobiopterin 188 Thought disorder 13 Thymopsyche 117,130 Thyroid autoimmunity 37 Topographical EEG analysis 153 TRH 1 Twin studies 13

Ultrasonography 37

Ventral tegmental area 96 Vigilance 42

Withdrawal 53

KARGEII C1997SKargerAG,

E-Mail karger@karger.ch Fax+ 41613061234 http://www.karger.ch 